\title{
Modulation Mode Recognition Based On Multi-Class Classification Of Support Vector Machine
}

\author{
Qian Ren \\ Department of Electronic Engineering \\ Beijing University of Technology \\ Beijing, 100124, China \\ e-mail: xiatian1096@126.com
}

\author{
Guangmin Sun, Yuanyuan Zhang \\ Department of Electronic Engineering \\ Beijing University of Technology \\ Beijing, 100124, China \\ e-mail: gmsun@bjut.edu.cn
}

\begin{abstract}
An analog and digital modulation recognition method based on support vector machine (SVM) is proposed. A multi-class classifier is designed through the reasonable use of SVM multi-class classification method. A comparison for the performances of one-against-all (OAA), one-against-one (OAO) and binary tree (BT) with the different kernels of SVM is made. Experimental results show that the Gaussian radial basis function (GRBF) kernel has better performance than others. It can be seen from the simulation result that the proposed method is correct and efficient. The scheme can achieve $93 \%$ recognition accuracy at low levels of SNR.
\end{abstract}

Keywords-Support vector machine; Modulation recognition; Multi-class classification

\section{INTRODUCTION}

With the great development of technology in communications, modulation types have become more and more complex. How to monitor and recognize the type of modulation efficiently is an important problem.

Generally, modulation recognition methods can be classified into two types: decision-theoretic (DT) approach [1,2] and pattern-recognition (PR) approach [3]. Probability and hypothesis testing arguments are used to formulate the recognition problem in DT methods. Support Vector Machine has powerful ability in pattern recognition, and it is widely used in modulation recognition with its better stability and mistake tolerance.

In the paper, the aim is to recognize simultaneous analog and digital modulation types (total 8 kinds of signals). In order to investigate the performances of three kinds of multi-class support vector machine classifiers (OAA, OAO and BT), six features for 8 types of modulated signals (AM, FM, 2PSK, 8PSK, 2FSK, 4FSK, 16QAM and 64QAM) are extracted and used for classification.

In the paper, the extraction method of six features is presented in Section II. The principle of Support Vector Machine (SVM) and four kinds of common Multi-class classification method are described in Section III, which include one-against-all (OAA), one-against-one (OAO), binary tree (BT) and Directed Acyclic Graph (DAG). In section IV, modulation recognition model is described. The results of the three methods (OAA, OAO and BT) with different kernels are discussed in Section V. Finally, some conclusions and recommendations for future work are presented.

\section{THE FEATURE EXTRACTION}

Feature extraction is the key for pattern recognition since the best classifier will perform poorly if the features are not chosen well. So it is arguably the most important component of an intelligent system designed for pattern recognition. Feature used as modulation classification must be selected so that they are sensitive to the modulation types of interest. In this paper, six features are chosen to classify AM, FM, 2PSK, 8PSK, 2FSK, 4FSK, 16QAM and 64QAM, which are $\boldsymbol{y}_{\text {max }}, \boldsymbol{\sigma}_{a p}, \boldsymbol{\sigma}_{a}, \boldsymbol{\sigma}_{a \boldsymbol{a}}, \boldsymbol{\sigma}_{\text {af }}$ and $\boldsymbol{K} \boldsymbol{a}$.

The first feature $\boldsymbol{V}_{\max }$ [4] is the maximum value of the spectral power density of the normalized-centered instantaneous amplitude, and it is defined by:

$$
\gamma_{\max }=\max \left|\operatorname{DFT}\left(a_{c n}(i)\right)\right|^{2} / N_{s}
$$

Where,

$$
\begin{gathered}
a_{c n}(i)=a_{n}(i)-1 \\
a_{n}(i)=\frac{a(i)}{m_{a}} \\
m_{a}=\frac{1}{N_{s}} \sum_{i=1}^{N_{s}} a(i)
\end{gathered}
$$

$N_{s}$ denotes the number of the samples, $m a$ is the Average amplitude of every frame.

The second feature, $\boldsymbol{\sigma}_{a p}$ is the standard deviation of the absolute value of the zero center non-weak section momentary phase nonlinear component, and it is defined by: 


$$
\sigma_{a p}=\sqrt{\frac{1}{C}\left(\sum_{a_{n}(t)>a_{t}} \phi^{2}(i)\right)-\left(\frac{1}{C} \sum_{a_{n}(i)>a_{t}}\left|\phi_{N L}(i)\right|\right)^{2}}
$$

where,

$$
\phi_{N L}(i)=\phi(i)-\phi_{0}=\phi_{N L}(i)-\frac{1}{N_{s}} \sum_{i=1}^{N_{s}} \phi(i)
$$

Here, $\boldsymbol{a}_{\boldsymbol{t}}$ denotes the amplitude circumscription level to judge non-weak section momentary of the signal, $\boldsymbol{c}$ denotes the number of the non-weak section momentary phase nonlinear component in all samples $\boldsymbol{N}_{s} \cdot \boldsymbol{\varphi}{ }_{N L}(i)$ denotes the zero center normalization momentary phase nonlinear components. In carrier synchronization conditions we can get such equation. $\boldsymbol{\varphi}(\boldsymbol{i})$ denotes momentary phase.

The third feature, the standard deviation of the normalized-centered instantaneous amplitude in the nonpeak segment of a signal $\boldsymbol{\sigma}_{\mathbf{a}}$, and it is defined by

$$
\sigma_{a}=\sqrt{\frac{1}{C}\left[\sum_{A_{n}(i)>a_{t}} A_{c n}^{2}(i)\right]-\frac{1}{C}\left[\sum_{A_{n}(i)>a_{t}} A_{c n}(i)\right]^{2}}
$$

Where, $\boldsymbol{f}_{\boldsymbol{s}}$ is the sampling rate. $\boldsymbol{A}_{\boldsymbol{c} n}(\boldsymbol{i})$ is the normalized instantaneous amplitude at time $L$ is the number of samples in $\left\{\boldsymbol{A}_{\boldsymbol{c}}(\boldsymbol{i})\right\}$ for which $\boldsymbol{A}_{\boldsymbol{n}}(\boldsymbol{i})>\boldsymbol{a}_{\boldsymbol{t}}$, and $\boldsymbol{a}_{\boldsymbol{t}}$ is a threshold for $A(t)$ below which the band-limitation is more affected on the instantaneous amplitude of the band-limited 2-PSK and the band-limited 4-PSK signals.

The fourth feature, Standard deviation of the absolute value of the zero center normalization momentary amplitude $\boldsymbol{\sigma}_{a a}$

$$
\sigma_{a a}=\sqrt{\frac{1}{N_{s}}\left(\sum_{1}^{N_{s}} a_{c n}^{2}(i)\right)-\left(\frac{1}{N_{s}} \sum_{1}^{N_{s}}\left|a_{c n}(i)\right|\right)^{2}}
$$

The fifth feature $\sigma$ af is the standard deviation of the absolute value of the zero center normalization non-weak section momentary frequency nonlinear components, and it is defined by:

$$
\sigma_{a f}=\sqrt{\frac{1}{C}\left(\sum_{a_{n}(i)>a_{t}} f_{N}^{2}(i)\right)-\left(\frac{1}{C} \sum_{a_{n}(i)>a_{t}} \mid f_{N}(i)\right)^{2}}
$$

The sixth feature $\boldsymbol{k} \boldsymbol{a}$ [5] is a parameter of the distinction between analog and digital modulation. We first assume that the receive sequence $X(i), i=1,2, \ldots, n$, so

$$
k a=\frac{E(X-\bar{X})}{\sigma^{4}}-3
$$

Here, $\boldsymbol{E}(\cdot)$ denotes the mean. $\boldsymbol{\sigma}^{4}$ denotes the square of the variance of $\boldsymbol{X}$.

\section{SUPPORT VECTOR MACHINE}

Support Vector Machine (SVM) is a useful technique for data classification and is considered to be the state-of-the-art tool for linear and nonlinear classification .It realizes classification tasks for two-class problem by using the separating hyperplane [6].The hyperplane is found by estimating the maximum distance to the closest data points of the training set. These closest data points are named support vectors (SVs). Data points can be transformed into a high dimensional space (HDS) by using a nonlinear transformation if they are not linearly separable in the input space. HDS is called feature space. These nonlinear transformations are represented by using kernel functions .It usually has five types: Linear, Polynomial, RBF, Sigmoid and Wavelet. The data points in the feature space are divided by the optimal separating hyperplane estimated by using the maximum distance to the closest data points of the training set mentioned as above.

The support vector machine (SVM) is used to solve two-class learning problems. Many SVM algorithms were introduced. Nonetheless, real-world classification tasks require more than two-class. Meanwhile, large margin separators have been resulted in binary problems, they can also be adapted to treat multi-class problems. In fact, there are many ways to solve Multi-class classification problems for SVM such as Directed Acyclic Graph (DAG), Binary Tree (BT), One-Against-One (OAO) and One-Against-All (OAA) classifiers.

\section{A. One-Against-All (OAA) SVM}

The approach "One-Against-All" is the simplest and oldest method of decomposition. The initial formulation of the one-against-all method states that a data point would be classified under a certain class if and only if that class's SVM accepted it and all other classes' SVMs rejected it. While accurate for tightly clustered classes, this method leaves regions of the feature space undecided where more than one class accepts or all classes reject [7].

\section{B. One-Against-One (OAO) SVM}

Another decomposition method is "One-Against-One", also known as "pairwise coupling", "all pairs" or "round robin" [8].This method consists of building an SVM for each pair of classes, or $\boldsymbol{c}(\boldsymbol{c}-1) / 2$ SVM for a problem with $\boldsymbol{C}$ classes. Each classifier is trained to separate the data from one class to those of another class. The decision rule is generally the majority voting called "max-wins voting". Each SVM votes for a class and the unknown data is finally associated with the class receiving most of the votes $[9,10]$. 
More advanced methods include using decision graphs to determine the class selected in a similar manner to knockout tournaments [11].

\section{Binary Tree of SVM (BTSVM) [12]}

This technique deploys multiple SVMs arranged in a binary tree structure. Every SVM in its related node of the tree is trained by using two of the classes. The measurement of similarity between the two classes and the remaining samples is made by deploying probabilistic outputs. All existing samples in the node are allocated to the two consequent subnodes of the previously chosen classes by similarity and this step iterates at every node until all samples of each node belong to one class. The drawbacks of this technique are high training time and low performance in huge training data sets; because all samples of every node should be tested to define their classes during constructing trees.

\section{Directed Acyclic Graph SVM (DAGSVM)}

Directed Acyclic Graph SVM is introduced by Platt [13], it works in training stage by constructing $M \times(M-1) / 2$ binary classifiers. In recognition stage, it uses a rooted binary directed acyclic graph which includes $M$ leaves and $M \times(M-1) / 2$ nodes. For a test sample, evaluation of binary decision functions starts at the root node; afterward its movement to either left or right side depends on the output value.

\section{CLASSIFIER}

Modulation identification includes inter-class classification and intra-class classification. In this paper, inter-class recognition is a simple two class classification problem based on SVM, and intra-class classification is a multi-class classification problem. The structure of the hierarchical classifier is as shown in 错误! 未找到引用 源。. Firstly, we extract seven features. Secondly, enter in the first-level classifier that can distinguish an analog or digital modulation mode. Thirdly, enter in the third-level classifier if it is the analog modulation, and enter in the second-level classifier if it is the digital modulation. Actually, SVM-2 is a classifier for digital modulation. In this step, the three digital modulations includes M-QAM, M-FSK and M-PSK Classification. Aiming at comparing the performances of OAA, OAA and BT for intea-class classification, we use the three multi-class classifications to identify them. Finally, enter in the third-level classifier. In this level, the modulation signal is a simple two-class classification problem.

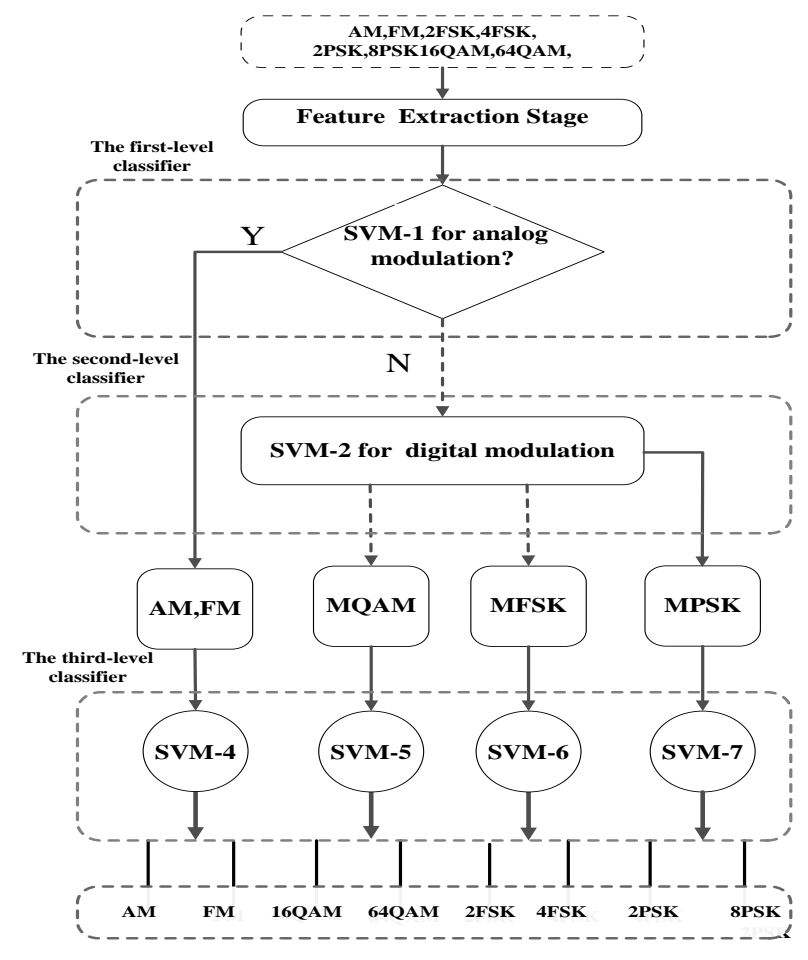

Figure 1. The structure of the hierarchical classifier

\section{CONCLUSION}

In this paper, the signal collected is from the Agilent E4438C ESG Vector Signal Generator. The SNR is below $0.2 \mathrm{~dB}$.The carrier frequency $\left(\boldsymbol{f}_{\boldsymbol{c}}\right)$ is $2 \mathrm{GHZ} \mathrm{kHz}$. Sampling rate $\left(f_{s}\right)$ is $80 \mathrm{kHz}$. The data rate is $24.3 \mathrm{~kb} / \mathrm{s}$.We use the LIBSVM simulation tool for the signal simulation. It is a simple and easy-to-use support vector machine tool for classification (C-SVC, nu-SVC), regression (epsilon-SVR, nu-SVR), and distribution estimation.

\section{A. The First-level Classifier.}

The recognition rate of Analog modulation is $100 \%$ through simulation experiments.The and digital modulation is also $100 \%$. As we all known, How to select the two parameters $\mathrm{C}$ and $\sigma 2$ is the key of classification. The LIBSVM use Cross validation to find the best parameters.

\section{B. The Second-level Classifier.}

In this step, we first use OAA to classify the three signals. The result is as shown in Table 1 . We can see the accuracy of the kernel function RBF and Linear are better than Polynomial and Sigmoid. The highest accuracy of M-QAM is below 90\%. In Table 2, we can see kernel function RBF is the best. All signal accuracy is more than $92 \%$. Meanwhile, the accuracy of M-FSK is $100 \%$. At last, in Table 3, the RBF is also best in the four kernel function. And, the accuracy of binary tree and OAO are the same. The two parameters $C$ and $\boldsymbol{\sigma}^{2}$ are decided by the LIBVIEW. 


\section{The Third-level Classifier.}

In this step, the four Classifier is designed as Fig 1 shown. SVM is a good tool for solving the two-level classification. It is shown that the accuracy of AM and FM are $100 \%$ in the SVM-4. Meanwhile, the accuracy of 2FSK, 4FSK, 2PSK, 8PSK, 16QAM and 64QAM are 100\%. And the best recognition result was drawn from the above work. It is shown in Table 4.

This paper presents Multi-class SVM design details and provided experimented results on a hierarchical SVM classifier. According the experiment, the RBF kernel is a reasonable first choice for inter-class and intra-class recognition. In this condition, the experimental results have shown the binary tree SVM and OAO SVM has the same recognition rate. But in practical application, the calculation steps of OAO SVM are more Complex than binary tree SVM. So the binary tree is the best choice for modulation recognition. In this paper, the recognition rate of the 8 types ((AM, FM, 2PSK, 8PSK, 2FSK, 4FSK, 16QAM and 64QAM) of modulated signals is above $93 \%$ at low levels of SNR.

TABLE I.

THE RegCognition Results Of OAA With THE DifFERERNT KeRNELS

\begin{tabular}{cccc}
\hline kernels & MPSK & MFSK & MQAM \\
\hline RBF & $94.118 \%$ & $100 \%$ & $87.5 \%$ \\
Linear & $94.118 \%$ & $100 \%$ & $81.25 \%$ \\
polynomial & $97.176 \%$ & 0 & $81.25 \%$ \\
Sigmoid & $55.882 \%$ & $95.238 \%$ & $37.5 \%$ \\
\hline
\end{tabular}

TABLE II.

THE REGCOGNITION RESUlts OF OA0 With THE DIFFERERNT KeRNELS

\begin{tabular}{cccc}
\hline kernels & MPSK & MFSK & MQAM \\
\hline RBF & $97.059 \%$ & $100 \%$ & $93.75 \%$ \\
Linear & $50 \%$ & $100 \%$ & 0 \\
polynomial & $97.059 \%$ & $100 \%$ & 0 \\
Sigmoid & $50 \%$ & $100 \%$ & 0 \\
\hline
\end{tabular}

TABLE III.

The RegCognition Results Of Binary Tree With The DifFERERnt KeRnELS

\begin{tabular}{cccc}
\hline kernels & MPSK & MFSK & MQAM \\
\hline RBF & $97.059 \%$ & $100 \%$ & $93.75 \%$ \\
Linear & $50 \%$ & $100 \%$ & $91.667 \%$ \\
polynomial & $97.059 \%$ & 0 & $97.917 \%$ \\
Sigmoid & $52.941 \%$ & $36.508 \%$ & $50 \%$ \\
\hline
\end{tabular}

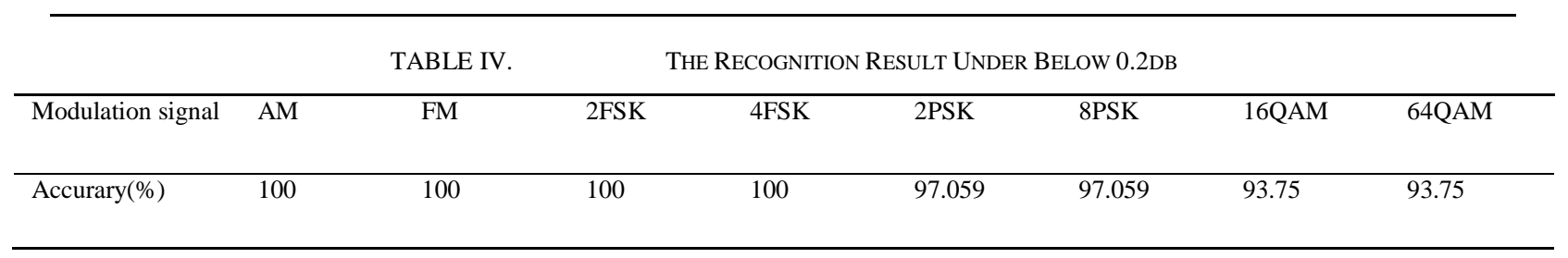

\section{REFERENCES}

[1] Nandi A K, Azzouz E E, "Automation modulation recognition," Signal Processing, vol. 46, May 1995, pp.211- 222.
[2] Ananthram Swami, Brian M. Sadler, " Hierarchical Digital Modulation Classification Using Cumulants," IEEE Transactions on Communication, vol. 48, Mar. 2000, pp.416- 429.

[3] E. E. Azzouz, A. K. Nandi, "Automatic modulation recognition-II," J. Franklin Inst, vol.334B, 1997, pp.275-305. 
[4] E. E. Azzouz, A. K. Nandi. Automatic identification of digital modulation types. Signal Processing, 1995, 47:55-69.

[5] Luna, study of Modulation Identification and Signals Feature Extraction 2008, Xidian University.

[6] Yao, Y., Frasconi, P., \& Pontil, M. (2001). Fingerprint classification with combinations of support vector machines. InAVBPA 2001, LNCS 2091 (pp. 253-258).

[7] Kallas, M., et al. Multi-Class SVM Classification Combined with Kernel PCA Feature Extraction of ECG Signals. In Telecommunications (ICT), 2012 19th International Conference on. 2012. Jounieh.

[8] J. Milgram, M. Cheriet, and R. Sabourin, "One Against One or One Against All: Which One is Better for Handwriting Recognition withSVMs?" inTenth International Workshop on Frontiers in Handwriting Recognition, G.Lorette, Ed., Universit'e de Rennes 1. La Baule (France): Suvisoft, October 2006.

[9] A. Pronobis and B. Caputo, "Confidence-based cue integration for visual place recognition," inin Proc. IROS07 Centre for Autonomous Systems Royal Institute of Technology, SE-100 44 Stockholm, Sweden, 2007.

[10] J. Fu, C. Huang, and S. Lee, "A multi-class svm classification system based on methods of self-learning and error filtering," Taiwan, Republic of China, 2008.

[11] C. Burges, "A tutorial on support vector machines for pattern recognition," Data Mining and Knowledge Discovery, vol. 2, pp. 121-167, June 1998.

[12] Chamasemani, F.F. And Y.P. Singh. Multi-class Support Vector Machine (SVM) classifiers - An Application in Hypothyroid detection and Classification. in Bio-Inspired Computing: Theories and Applications (BIC-TA), 2011 Sixth International Conference on. 2011. Penang.

[13] Campbell, W. M., Singer, E., Torres-Carrasquillo, P. A., \& Reynolds, D. A. (2004).Language recognition with support vector machines, Odyssey, Toledo, Spain. E. E. Azzouz, A. K. Nandi. Automatic identification of digital modulation types. Signal Processing, 1995, 47:55-69 